\title{
The distribution of ticks of the Ornithodoros moubata complex (Ixodoidea: Argasidae) in Malawi, and its relation to African swine fever epizootiology
}

\author{
BY J.M. HARESNAPE* AND F. D. MAMU \\ Central Veterinary Laboratory, Lilongwe, Malawi
}

(Received 9 September 1985 ; accepted 29 October 1985)

\begin{abstract}
SUNIIARY
The distribution of ticks of the Ornithodoros moubata complex in different habitats in Malawi, particularly pigsties and houses, was established from a four-phase survey undertaken between 1982 and 1985. The first phase consisted of preliminary interviews, the second phase consisted of a questionnaire to Veterinary Assistants (VAs) in rural areas, the third involved tick collections by VAs and the fourth involved both interviews and tick collections by members of the Central Veterinary Laboratory, Lilongwe. The area in which O. moubata is found in pigsties includes much of the African swine fever (ASF) enzootic area, and it seems likely that the enzootic area could become larger in future. The occurrence of $O$. moubata in warthog habitats was also investigated.
\end{abstract}

\section{INTRODUCTION}

The soft tick Ornithodoros moubata, or eyeless tampan, is important in the epidemiology of relapsing fever of humans (Walton, 1962) and African swine fever (ASF) of pigs (Plowright, Parker \& Pierce, 1969). It is because of its role as a vector of ASF that this investigation was initiated.

The occurrence of $O$. moubata in houses in most countries in Africa including Malawi is well documented (Leeson, 1953; Hoogstraal, 1956; Walton, 1962, 1964) as is its occurrence in warthog burrows (Hoogstraal, 1956; Plowright et al. 1969; Thomson et al. 1983). It has also been reported in pigsties in Angola (Wellman, 1906, 1907), Zimbabwe (Jack, 1921), Zaire (Schwetz, 1927) and Ruanda-Burundi (Jadin, 1951) and has been found associated with domestic pigs in Malawi (Wilson, 1943) and South Africa (Bedford, 1936). There are various reports of other domestic hosts, but Hoogstraal (1956) suggests the pig to be the most important.

The aims of this study were to delineate the distribution of tampans in both pigsties (kholas) and houses throughout Malawi, to compare their presence in pig kholas with the areas of Malawi where ASF is enzootic, and to establish whether they also occur associated with warthogs in Malawi.

* Present address: 2 Southcott, Pewsey, Wiltshire, UK. 


\section{METHODS}

Since the tampan gives a painful bite and transmits relapsing fever, people in villages where it inhabits houses are fully aware of its presence and the first two phases of this survey took advantage of this source of information. The presence of tampans in pig kholas is less obvious and could only be established in the third and fourth phases of the survey, from more thorough investigations.

In the first phase, 78 members of the Veterinary Department stationed in Lilongwe, including at least one originating from each of the 24 districts of Malawi, were interviewed. Each was shown some live tampans and asked if he had ever seen them in his home district, in houses or elsewhere, in order to obtain preliminary information about where they were most common.

In the second phase, questionnaires were sent to all 348 Veterinary Assistants (VAs) stationed in rural areas throughout Malawi. Since these officers supervise veterinary activities such as dipping cattle, they have a close working relationship with the farmers in their dipping areas and are ideally situated to collect information from villagers. Each VA was asked if he had seen tampans (locally known as nkhufi) in his area, and if so whether in houses, pig kholas or elsewhere; whether in the roof, walls or floor; whether in mud, wood, brick or other material, and whether he had seen the same tick in any other part of Malawi such as his home district or any other place he had been. In a separate questionnaire, each VA was asked to estimate the domestic pig population in his area, and from this the domestic pig population and pig density was estimated for each district (Table 1).

In the third phase, one specimen of $O$. moubata was sent in a sealed container to each District Veterinary Office to be shown to all VAs in the district. Empty plastic containers were also distributed, and all VAs were requested to collect tampans, to record the type of premises in which they had been found (house, pig khola or other), and to return them to the Central Veterinary Laboratory (CVL).

Finally, visits were made to some areas to look for and collect tampans in the fourth phase. Although it was not possible to cover the entire country, a great many areas in the Central and Southern Regions of Malawi were visited by staff of the CVL during 1983 and 1984 during an ASF serological survey (Haresnape, Lungu \& Mamu, 1985). At the same time, searches were made for tampans in both houses and pig kholas in each area visited. This was particularly useful in areas where they were not well known, because it allowed their reported absence from pig kholas as well as from houses to be verified. Specimen tampans were carried for demonstration and, in areas where the villagers said they did occur but not in the immediate vicinity, the VA was asked to make a more thorough search in the areas indicated. Extra visits were made to areas of particular interest such as those where the VA reported the presence of tampans in pig kholas as well as in houses, and to those areas in which many pigs are kept but where the VA reported that tampans were absent from pig kholas.

Specimen tampans from 10 different districts, including at least 10 from each and more than 100 from most, were sent to the Animal Virus Research Institute, Pirbright, U.K. for identification. Wherever possible tampans from both pig kholas and houses were sent. 
Table 1. Results of the second phase of the tampan survey and pig census

\begin{tabular}{|c|c|c|c|c|c|c|}
\hline \multirow[b]{2}{*}{ District } & \multirow[b]{2}{*}{$\begin{array}{l}\text { Proportion of } \\
\text { questionnaires } \\
\text { returned }\end{array}$} & \multicolumn{3}{|c|}{ Tampans in houses } & \multirow{2}{*}{$\begin{array}{l}\text { Domestic } \\
\text { pig } \\
\text { population } \dagger \\
\text { (approx) }\end{array}$} & \multirow{2}{*}{$\begin{array}{c}\text { Average } \\
\text { pig } \\
\text { density } \ddagger \\
\left(\text { pigs } / \mathrm{km}^{2}\right)\end{array}$} \\
\hline & & Present* & Absent* & $\begin{array}{l}\text { Don't } \\
\text { know }\end{array}$ & & \\
\hline $\begin{array}{l}\text { Northern Region } \\
\text { Chitipa (CH) } \\
\text { Karonga (KR) } \\
\text { Rumphi (RU) } \\
\text { Mzimba (MZ) } \\
\text { Nkhata Bay (NB) }\end{array}$ & $\begin{array}{c}21 / 23 \\
18 / 21 \\
4 / 9 \\
44 / 49 \\
4 / 5\end{array}$ & $\begin{array}{c}1(1) \\
0 \\
2 \\
43(43) \\
2(1)\end{array}$ & $\begin{array}{c}19(10) \\
17(5) \\
1(1) \\
1(1) \\
2(1)\end{array}$ & $\begin{array}{l}1 \\
1 \\
1 \\
- \\
-\end{array}$ & $\begin{array}{r}1500 \\
3200 \\
1000 \\
13100 \\
100\end{array}$ & $\begin{array}{l}0.5 \\
1 \cdot 1 \\
0.5 \\
1 \cdot 5 \\
0.03\end{array}$ \\
\hline $\begin{array}{l}\text { Central Region } \\
\text { Nkhotakota (NK) } \\
\text { Kasungu (KS) } \\
\text { Ntchisi (NT) } \\
\text { Dowa (DO) } \\
\text { Mchinji (NC) } \\
\text { Lilongwe (LL) } \\
\text { Salima (SA) } \\
\text { Dedza (DE) } \\
\text { Ntcheu (NC) }\end{array}$ & $\begin{array}{l}1 / 3 \\
7 / 18 \\
2 / 6 \\
7 / 18 \\
5 / 6 \\
12 / 44 \\
6 / 9 \\
18 / 23 \\
19 / 21\end{array}$ & $\begin{array}{l}0 \\
3(1) \\
2(1) \\
6(1) \\
3 \\
9 \S(1) \\
1 \\
4(1) \\
0\end{array}$ & $\begin{array}{l}1 \\
3 \\
0 \\
1 \\
2(2) \\
3 \\
5(1) \\
12(1) \\
19(2)\end{array}$ & $\begin{array}{l}- \\
- \\
- \\
- \\
-\end{array}$ & $\begin{array}{r}1000 \\
11000 \\
3700 \\
7600 \\
19000 \\
30200 \\
2900 \\
16800 \\
20700\end{array}$ & $\begin{array}{l}0 \cdot 5 \\
3 \cdot 0 \\
2 \cdot 5 \\
2 \cdot 7 \\
7 \cdot 7 \\
6 \cdot 6 \\
1 \cdot 8 \\
5 \cdot 4 \\
6 \cdot 6\end{array}$ \\
\hline $\begin{array}{l}\text { Southern Region } \\
\text { Mangochi (MIN) } \\
\text { Machinga (MA) } \\
\text { Zomba (ZO) } \\
\text { Mwanza (MIV) } \\
\text { Blantyre (BT) } \\
\text { Chiradzulu (CD) } \\
\text { Mulanje (ML) } \\
\text { Thyolo (TH) } \\
\text { Chikwawa (CK) } \\
\text { Nsanje (NS) }\end{array}$ & $\begin{array}{l}1 / 3 \\
5 / 9 \\
3 / 11 \\
4 / 6 \\
6 / 11 \\
10 / 11 \\
7 / 12 \\
1 / 7 \\
5 / 13 \\
6 / 10\end{array}$ & $\begin{array}{l}0 \\
1 \S \\
0 \\
1 \\
0 \\
0 \\
3 \S(1) \\
1 \\
0 \\
1\end{array}$ & $\begin{array}{l}0 \\
3 \\
2(1) \\
3 \\
6 \\
10 \\
2 \\
0 \\
3(2) \\
5\end{array}$ & $\begin{array}{l}1 \\
1 \\
\frac{1}{-} \\
\frac{2}{2} \\
\frac{2}{-}\end{array}$ & $\begin{array}{r}400 \\
800 \\
3400 \\
3600 \\
7400 \\
2700 \\
12300 \\
5200 \\
15500 \\
6000\end{array}$ & $\begin{array}{l}0 \cdot 1 \\
0 \cdot 2 \\
1 \cdot 6 \\
1 \cdot 9 \\
4 \cdot 2 \\
3 \cdot 9 \\
4 \cdot 5 \\
3 \cdot 6 \\
5 \cdot 2 \\
4 \cdot 6\end{array}$ \\
\hline
\end{tabular}

Brief searches were made for tampans in warthog habitats in three National Parks, one in each Region. Warthog-associated tampans were also sent to Pirbright for identification.

The ASF enzootic area was taken to be that in which seropositive domestic pigs had been identified as reported previously (Haresnape et al. 1985).

\section{RESULTS}

The preliminary interviews (first phase) indicated striking differences between districts within MIalawi, the tampan being particularly well-known in Mzimba district in the Northern Region and Mchinji district in the Central Region. Of the 78 people interviewed, 24 had seen tampans recently in their home district, all in 


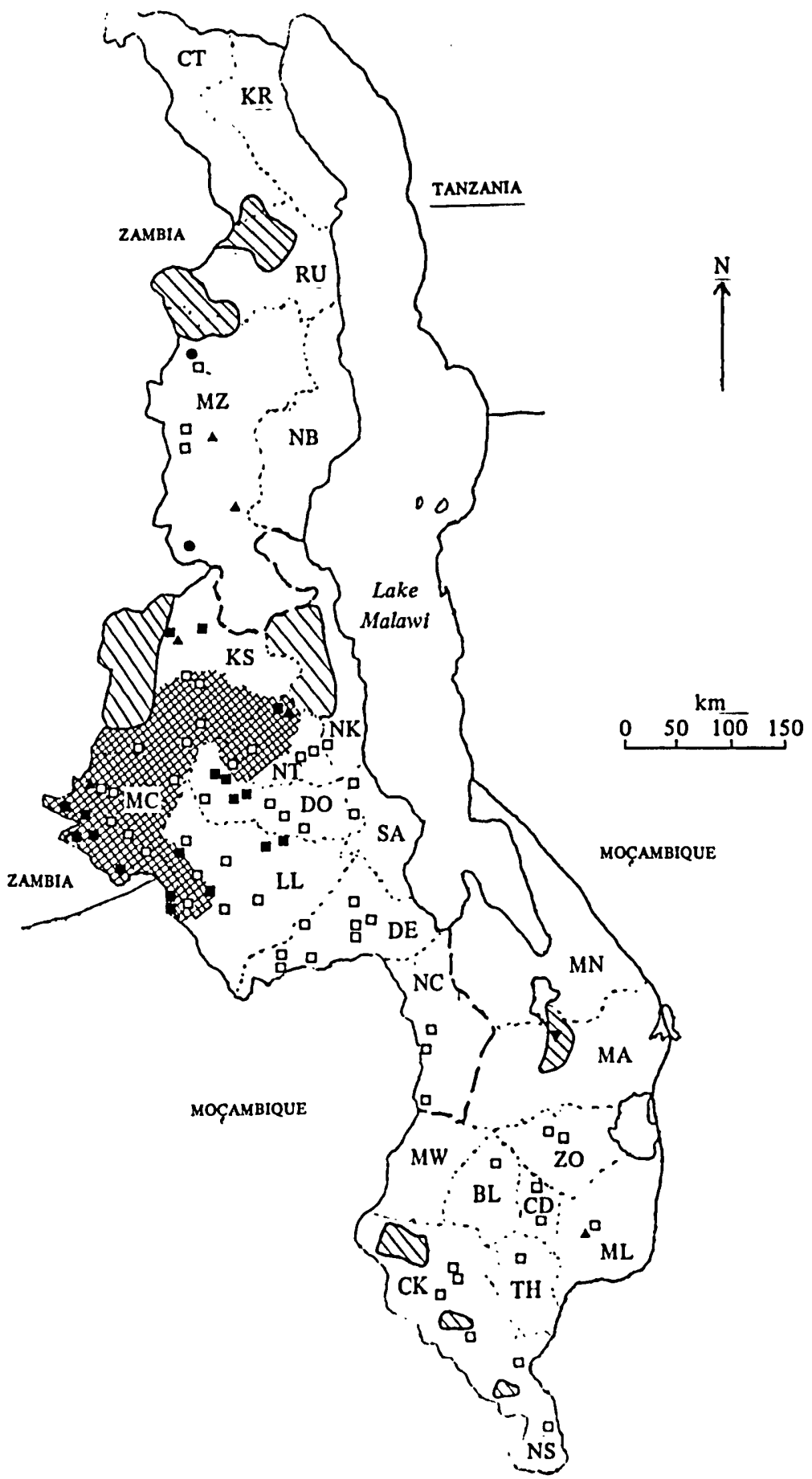

Fig. 1. Distribution of Ornithodoros moubata in pig kholas and warthog habitats in Malawi.

QJPNational Park or Game Reserve.

Known $\triangle \mathrm{SF}$ enzootic area (Haresnape el al. 1985).

$O$. moubata present in pig kholas: $O$, convincing report (second phase of survey); $\Delta$, received by post (third phase); $\square$, collected by CVL staff (fourth phase). $O$. moubata absent from pig kholas: $\square$, searches made by CVI staff (fourth phase). O. moubata found in warthog habitats: $\nabla$, collected by CVI, staff. CT, Ietters refer to districts

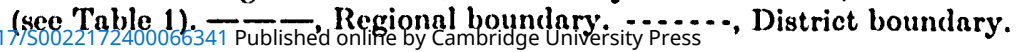


the Northern and Central Regions. Seven could remember seeing them in the past but not for several years. It is important to note that all eight people from Mzimba district and all seven people from Mchinji district who were interviewed reported presence of tampans. The only report from the Southern Region was from a man from Mwanza district who recalled seeing them 20 years ago. The differences found in the first phase of the survey were followed up in the second, third and fourth phases, the results of which are summarized below and in Table 1 and Figs. 1 and 2.

\section{Northern Region}

The response to the questionnaire (second phase of the survey) was particularly good from the Northern Region, 91 of the 107 questionnaires distributed being completed and returned (Table 1). Forty-three of the $44 \mathrm{VAs}$ from Mzimba district who replied reported tampans to be common in houses in their dipping areas. Convincing reports of the presence or absence of tampans in houses (Fig. 1) were therefore taken to be those from VAs who reported having seen tampans in Mzimba district, since both the first and second phases of the survey indicated that they were well known there. For example, $36 \mathrm{VAs}$ from Karonga and Chitipa districts reported absence of tampans from their areas. Fifteen of these VAs had seen tampans in Mzimba district, and this was therefore taken to be convincing evidence for the absence of tampans in their 15 areas. Only one convincing report of the presence of tampans in the Northern Region outside Mzimba district was obtained in this second phase, and this was from the Nkhata Bay district.

Twenty-seven VAs in the Northern Region sent tampans from houses to the CVL (third phase). Twenty-five of these were from Mzimba district, one was from Rumphi district and one was from Nkhata Bay district sent by the VA covering Likoma and Chizumulu Islands. Most were from VAs who had already given a convincing report of tampans in houses in the questionnaire.

There were only two convincing reports of tampans in pig kholas in the Northern Region, and only two VAs sent tampans from pig kholas to CVL. The density of domestic pigs is generally lower in the Northern Region than elsewhere in Malawi ('Table 1), primarily because of cultural differences, and this is the most probable reason why few tampans could be found in pig kholas. The fourth phase of the survey did not cover this Region because of the low pig density and because ASF has not been reported there.

No tampans were found during a brief search in warthog burrows in Nyika National Park in Rumphi district.

\section{Central Region}

Convincing reports of the presence of tampans in houses were obtained in the second phase from five VAs ('Table 1 and Fig. 2). Such reports were again taken as being those from VAs who reported having seen tampans in Mzimba district. However most of the information for the Central Region was obtained during the third and fourth phases (Figs. 1 and 2).

In the third phase, $24 \mathrm{VAs}$ sent tampans from houses and $3 \mathrm{VAs}$ sent tampans from pig kholas to the CVL. None was sent from any other type of premises, and most arrived in good condition. One VA sent lice instead of tampans and these were ignored. 




Fig. 2. Distribution of Ornithodoros moubala in houses in Malawi. National Park or Game Reserve.

o. moubata present in houses: $\boldsymbol{O}$, convincing report (second phase of survey); $\Delta$, received by post (third phase); ${ }^{-}$, collected by CVI staff (fourth phase). $O$. moubala absent from houses: $O$, convincing report (second phase); $\square$, searches made by $C V L$ staff (fourth phase). CT, Letters refer to districts (seo Table 1). - - Regional boundary. ......... District boundary. 
The fourth phase was used to build on the information obtained during the third phase, particularly in areas where the data were considered to be inadequate. Information given by villagers on the presence or absence of tampans in houses was found to be very reliable. However, in general, the local people were unaware of their presence in pig kholas, even in areas where they were well-known in houses. Therefore particular effort was made during this final phase to search in pig kholas. No areas were discovered in which tampans inhabited pig kholas but not houses. Those localities where no tampans were found by CVL staff and in addition where the local people, after seeing the demonstration tampans, verified that they did not occur, are marked on Fig. 2 as areas where the tampan is absent. Areas where no tampans could be found in pig kholas are marked on Fig. 1.

The results show that tampans occur in the north of Kasungu district, in Mchinji, Lilongwe, Dowa and Ntchisi districts, and in the north part of Dedza district. The southern part of Kasungu district consists largely of tobacco estates and consequently has few villages. No tampans were found in the lakeshore area (Nkhotakota and Salima districts) or in Ntcheu district in the southern part of the Region. In most areas where tampans were present in both pig kholas and houses, they were easier to find and therefore presumably more numerous in houses. In general they were more commonly found in older premises, and were usually absent from newly built houses. The situation could differ tremendously even over short distances. For example, there was no trace of tampans in one group of villages in Lilongwe district although they were numerous in both houses and pig kholas in villages in Mchinji district on the opposite bank of the Namitete river just $3 \mathrm{~km}$ away.

No tampans were found during a brief search in warthog burrows in Kasungu National Park.

\section{Southern Region}

Only one convincing report of the presence of tampans in this Region was obtained from the questionnaire (second phase) and this was in Mulanje distict. It appeared from the replies that some VAs were confusing tampans with insects such as lice or bedbugs and these reports were ignored. One VA reported presence of tampans in a goat khola in Mwanza district, but was unable to collect any from these premises to send to the CVL. Three VAs sent tampans from houses, and one sent tampans from pig kholas to CVL in the third phase. All arrived in good condition except one batch of three tampans from a house in Mwanza district which were dead on arrival. No tampans were found in the Southern Region by CVL staff during the fourth phase, despite searches in seven districts. No searches were made in Machinga or Mangochi districts, which are predominantly Moslem areas with few pigs, or in Mwanza district where the pig density is also low. Thus tampans are less well known, and presumably less widespread, in the Southern Region than elsewhere in Malawi but nevertheless do occur in parts of Mwanza, Thyolo and Mulanje districts.

In contrast, a great many tampans were found associated with warthogs in Liwonde National Park in Mangochi district. They were found in a sandy culvert known to be a warthog resting place, but absent in two adjacent culverts believed not to be frequented by warthogs. 
Extensive collections of tampans from houses and pig kholas in many areas and one large collection from a warthog resting place were made. These ticks were sent to Pirbright for identification and for ASF virus isolation tests. All were identified as being of the Ornithodoros moubata complex, and most of those from houses and pig kholas were $O$. porcinus porcinus (sensu Walton, 1962). The morphology and ASF status of the warthog-associated tampans has not been examined at the time of writing.

The ASF enzootic area is shown in Fig. 1 and is taken from a previous publication (Haresnape et al. 1985) with some additional areas such as central Kasungu district, which have been discovered to be enzootic more recently.

The domestic pig population is subject to considerable fluctuation particularly as a result of ASF outbreaks, and consequently the estimates of pig population and pig density (Table 1) can serve only as a rough guide.

\section{DISCUSSION}

The eyeless tampan, $O$. moubata, occurs in houses over a wide area of Malawi, being particularly well known in Mzimba district in the Northern Region, and is also widely distributed in the Central Region. It is less widespread in pig kholas and appears to be restricted to areas where the pig density is high, as in Michinji district. Where it is found in both types of premises, it is generally more common in houses. It therefore seems most likely that the tampans of Malawi have man as their primary host, and more rarely bite domestic pigs. How they move from one set of premises to another is not clear, but they could easily be transferred from houses to kholas in bedding, because it is common practice in many areas to air blankets by spreading them over pig kholas or other constructions. This seems a more likely route of transfer than on people, animals or poultry, because the ticks are generally only found on their hosts at night.

The areas of Malawi where the tampan infests pig kholas include much of the ASF enzootic area (Fig. 1). Although tampans are absent from pig kholas in some localities within the enzootic area, their presence within the area may be significant, as the tampan is a known vector of ASF (Plowright et al. 1969; Thomson et al. 1983). They may play an important role in the epizootiology of the disease in the Central Region of Malawi, presumably acting as a natural virus reservoir from which domestic pigs regularly become infected. ASF virus has been isolated at the Animal Virus Research Institute, Pirbright, from tampans collected within the enzootic area, and this will be the subject of a later paper.

It may be because there are few tampans associated with domestic pigs in the Southern Region that ASF has not become enzootic, despite a high pig density and numerous outbreaks in past years, for example in Chikwawa, Nsanje, Thyolo and Mulanje districts. Similarly, in the western part of Dedza district, ASF has not become enzootic although there have been numerous outbreaks in recent years (Haresnape, 1984; Haresnape et al. 1985); the only tampans found in this district were further north and only in houses. ASF has apparently not spread to the Northern Region where tampans are numerous, presumably because the pig density is low. However, the widespread occurrence of tampans outside the ASF enzootic area indicates that the enzootic area could become larger in future. Areas where tampans are found in domestic pig kholas are likely to be the most at risk, 
particularly those where ASF outbreaks have occurred recently such as in Dowa and Lilongwe districts (Haresnape et al. 1985).

Outside the enzootic area, most ASF outbreaks probably begin following the introduction of infected meat from an affected area. The mortality approaches $100 \%$ in outbreaks outside the enzootic area but is considerably lower in outbreaks within this area (Haresnape et al. 1985). The presence of $O$. moubata may be linked to this reduction in mortality, and current efforts are concentrated on trying to understand how this might be.

It has been suggested that altitude is an important limiting factor in the distribution of $O$. moubata in Kenya where it is present from sea level to about $2500 \mathrm{~m}$ and in Ethiopia where it is absent above about $2000 \mathrm{~m}$ (reviewed by Hoogstraal, 1956). Most areas of Malawi where tampans were found during this survey are between 1000 and $2000 \mathrm{~m}$, but the only extensive areas above this altitude are Nyika plateau in the Northern Region and Mulanje mountain in the Southern Region, which are largely uninhabited by man. No tampans were found during the brief search in warthog burrows in Nyika National Park. Except for Likoma and Chizumulu Islands, no tampans were found in the lakeshore area. Apart from these, and those associated with warthogs in Liwonde National Park, few were found below $1000 \mathrm{~m}$. In Ntchisi district they were numerous in houses on the plateau area at about $1400 \mathrm{~m}$, still present but less numerous on the escarpment above about $1100 \mathrm{~m}$ and totally absent from the lower escarpment and lakeshore areas. In Chikwawa and Nsanje districts, which are almost entirely below $500 \mathrm{~m}$, tampans were also totally absent. It appears that $O$. moubata may have been more widespread in the past because, in some areas, only the older people had ever seen them.

The occurrence of warthog-associated tampans in Liwonde National Park in the Southern Region is interesting because it is far away from any area where tampans have been found in houses or pig kholas. It is possible that the warthog-associated tampans are of a different sub-species and have different climatic and altitude requirements. Morphological comparisons are underway to investigate this possibility.

Warthog (Phacochoerusaethiopicus) \&re believed to play a role in the epizootiology of ASF in Malawi, since ASF antibodies have been detected in warthog sera from Lengwe National Park in Chikwawa district in the Southern Region (Haresnape et al. 1985). However, warthogs are rarely seen outside National Parks and Game Reserves (Hough, 1982) and consequently do not come into contact with domestic pigs, and warthog-associated ticks are perhaps less likely to be important in the epizootiology of ASF in domestic pigs than those inhabiting pig kholas.

We wish to thank the Secretary for Agriculture for permission to submit this work for publication and the Chief Veterinary Officer and his field staff, especially the many Veterinary Assistants who participated in the survey. Thanks are due to the staff of the Animal Virus Research Institute, Pirbright, UK, particularly to Dr P. Mellor and Mr R. Osborne for identifying the tampans and to Dr P. Wilkinson and his team for making virus isolations from them. The cooperation of the Ministry of Health, Malawi, and the Department of National Parks and Wildlife is also gratefully acknowledged. 


\section{REFERENCES}

Anonymous (1984). General land use and natural physical constraints. Report of the National Physical Development Plan MLW 79/012, p. 4. Lilongwe: Town and Country Planning Department.

BeDFond, G. A. H. (1936). A synoptic check-list and host-list of the ectoparasites found on South African Mammalia, Aves and Reptilia (Supplement no. 1). Onderstepoort Journal of Veterinary Science 7, 60-110.

Haresnape, J. M. (1984). African swine fever in Malawi. Tropical Animal Health and Production $16,123-125$.

Haresnape, J. M., Lungu, S. A. M. \& Mamu, F. D. (1985). A four-year survey of African swine fever in Malawi. Journal of Hygiene 95, 309-323.

HoogstranL, H. (1956). African Ixodidea. I. Ticks of the Sudan. United States Naval Medical Research Report N.M.005.050.29.07, pp. 121-190.

Hovan, J. Editor (1082). Mammals of Malawi, p. 30. Lilongwe: Environmental Unit, Department of National Parks and Wildlife.

$J_{A C K}, R$. W. (1921). Ticks infesting domestic animals in Southern Rhodesia. Rhodesian Agricullural Journal 18, 480-493.

JADIN, J. (1951). Contribution a l'étude des intoxications alimentaires au Ruanda-Urundi. Revue Belge de Pathologie et de Médicine Experimentale 21, 8-10.

LEeson, H.S. (1953). Some notes on the recorded distribution of old world species of Ornithodoros (Acarina). Bulletin of Entomological Research 44, 517-526.

Plowrioht, W., Parker, J. \& Pierce, M. A. (1069). The epizootiology of African swine fever in Africa. Veterinary Record 85, 668-674.

Schwetz, J. (1927). Note sur les Ixodidae (tiques) du Katanga. Revue Zoologique Africaine 15, 65-72.

Thomson, G., Gainaru, M., Lewis, A., Biggs, H., Nevill, E., van der Pypekamp, H., Gerber, L., Esterhuysen, J., Bengis, R., Bezuidenhout, D. \& Condy, J. (1983). The relationship between African swine fever virus, the warthog and Ornithodoros species in Southern Africa. In African Swine Fever: CEC/FAO Research Seminar, Sardinia, 1981 (ed. P. J. Wilkinson), pp. 85-100.

Walton, G. A. (1962). Aspects of Disease Transmission by Ticks. Symposium of the Zoological Society of London, no. 6 (ed. D. R. Arthur), pp. 83-156.

Walton, G. A. (1964). The Ornithodorus "moubata" group of ticks in Africa. Control problems and implications. Journal of Medical Entomology 1, 53-64.

Wellman, F. C. (1906). On Ornithodoros moubata, Murray, a disease-bearing African tick. Medicine (Detroit) 12, 493-499.

Wellanan, F. C. (1907). Prelimninary note on some bodies found in ticks - Ornithodoros moubata (Mfurray)-fed on blood containing embryos of Filaria perstans (Manson). British Medical Journal 2, 142-143.

Wrrson, S. G. (1943). Cattle ticks and their control by dipping in Nyasaland. Nyasaland Agricultural Quarterly Journal 3, 15-24. 